\title{
Competência de estudo para uma amostra universitária da área de exatas
}

\author{
Maria Cristina Rodrigues Azevedo Joly \\ Universidade de Brasília - DF \\ Diego Vinícius da Silva \\ Faculdade de Ciências Sociais Aplicadas de Extrema - MG \\ Carla Fernanda Ferreira-Rodrigues \\ Universidade São Francisco - SP \\ Juliana Maximila de Paula Bueno \\ Universidade São Francisco - SP \\ Leandro Silva Almeida \\ Universidade do Minho (Braga-Portugal)
}

\begin{abstract}
Resumo
Este estudo avaliou a percepção de 126 universitários sobre a utilização de estratégias de autorregulação de estudo. Os estudantes eram da área de exatas de uma faculdade isolada particular mineira, com idade entre 17 e 48 anos, e $81 \%$ deles eram do sexo masculino. Utilizou-se a Escala de Competência em Estudo (ECE-C\&T) para avaliar a percepção dos estudantes acerca de comportamentos estratégicos de planejamento, de monitoramento e de autoavaliação. Os alunos indicaram que a autorregulação de sua aprendizagem e as estratégias de planejamento de estudo são as mais utilizadas. As mulheres utilizaram mais estratégias de planejamento que os homens e não houve diferença estatisticamente significativa entre os cursos de exatas nem entre diferentes grupos de idade. Assim se conclui que, embora os alunos apresentem estratégias autorregulatórias, essas estão voltadas mais frequentemente para o planejamento do estudo, o que revela a necessidade de maior utilização de estratégias de monitoramento e autoavaliação para um estudo competente.
\end{abstract}

Palavras-chave: Autorregulação; hábitos de estudo; avaliação.

\section{Competency in study for a sample of the math and science area in the university}

\begin{abstract}
This article reports on the perception of 126 students on the use of self-regulation strategies to study. They studied in math and science area of a secluded private Minas Gerais college, aged between 17 and 48 years, and $81 \%$ were male. The Competence Scale in Study (ECE-C \& T) was used to assess the students' perception about strategic behavior of planning, monitoring and self-evaluation. The students self-regulated their learning and the planning of study strategies are the most used. Women used more planning strategies than men and there was no statistically significant difference between the math and science courses and age groups. It was found that, although the participants reveal they use selfregulatory strategies, these are often geared more for planning the study that indicates the need for greater use of monitoring strategies and self-assessment for competent study.
\end{abstract}

Keywords: Self-regulation; study habits; evaluation.

\section{Competencia de estudio para una muestra universitaria del área de exactas}

\section{Resumen}

Este estudio evaluó la percepción de 126 universitarios sobre la utilización de estrategias de autorregulación de estudio. Los estudiantes eran del área de exactas de una facultad aislada particular minera, con edad entre 17 y 48 años, y el $81 \%$ de ellos eran del sexo masculino. Se utilizó la Escala de Competencia en Estudio (ECE-C\&T) para evaluar la percepción de los estudiantes acerca de comportamientos estratégicos de planificación, demonitoreo y de autoevaluación. Los alumnos indicaron que la autorregulación de su aprendizaje y las estrategias de planificación de estudio son las más utilizadas. Las mujeres utilizaron más estrategias de planificación que los hombres y no hubo diferencia estadísticamente significativa entre los cursos de exactas ni entre diferentes grupos de edad. Así se concluye que, aunquelos alumnos presentenestrategias de autorregulación, esas están volcadas con más frecuencia a la planificación del estudio, lo que revela la necesidad de más utilización de estrategias demonitoreoy autoevaluación para un estudio de calidad.

Palabras-clave: autorregulación da aprendizaje; hábitos de estudio; evaluación. 


\section{Introdução}

Nas últimas três décadas aumentaram as investigações empíricas e reflexões teóricas acerca da autorregulação da aprendizagem, rentabilizando o significado deste construto para a compreensão dos processos de aprendizagem e do rendimento acadêmico dos estudantes. Ao integrar dimensões cognitivas, motivacionais, atitudinais e de conduta, a autorregulação da aprendizagem tornou-se um tema recorrente da investigação em psicologia educacional quando se pretende melhorar a qualidade das sua aprendizagem e contribuir para o seu sucesso acadêmico (Zimmerman, 2001; 2013).

A autorregulação remete ao processo de autodireção, no qual os alunos transformam suas habilidades cognitivas em tarefas relacionadas com as habilidades acadêmicas. Essa mudança é indicativa de uma aprendizagem efetiva que fornece sinais do desenvolvimento da competência do estudante (Zimmerman, 2001). Braun, Jackson e Wiley (2002) escreveram que os estudantes mais competentes utilizam conceitos relevantes para representar um problema e estratégias eficientes orientadas para as metas, automonitoram continuadamente seu aprendizado e apresentam justificativas coerentes para a explicação da resolução de problemas.

A análise comparativa realizada por Zimmerman (2001) sobre o processo de autorregulação da aprendizagem revelou que as definições tendem a variar de acordo com a perspectiva teórica. De modo geral, a autorregulação é definida como a habilidade dos alunos em gerenciar seus pensamentos, sentimentos e ações para atingir seus objetivos de aprendizagem. Refere-se, também, ao uso intencional de processos específicos ou estratégias para melhorar o desempenho acadêmico. Outra característica comum da maioria das definições de autorregulação é o feedback auto-orientado durante o aprendizado, que se refere a um processo cíclico em que os estudantes passam a monitorar a eficácia dos seus métodos de aprendizagem ou estratégias e responder a suas avaliações, como, por exemplo, a substituição de uma estratégia de aprendizagem por outra diante das exigências particulares de uma determinada tarefa ou contexto.

Para Rosário e cols. (2007), um dos desafios do Ensino Superior é promover um ensino de qualidade e levar os estudantes a desenvolverem os processos de aprendizagem. O padrão de ensino voltado para a transmissão de informações está sendo substituído progressivamente por outro mais orientado para o desenvolvimento de competências. Entende-se por competência de estudo a capacidade de adequar estratégias e criar técnicas de estudo, em um período de tempo, com a finalidade de obter sucesso na aquisição do conhecimento e no desempenho acadêmico.

Tavares e cols. (2003) realizaram um estudo com estudantes da Universidade dos Açores com o objetivo de identificar as estratégias, atitudes e procedimentos de estudo por meio da aplicação do Questionário de Atitudes e Comportamentos de Estudo. Participaram do estudo 370 alunos de quatro cursos de licenciatura existentes em dois pólos da universidade, entre os anos 2001 e 2002. Quanto à utilização de estratégias de autorregulação, identificaram que a maioria dos participantes utilizava mais estratégias cognitivas de planejamento e organização do estudo e estratégias cognitivas e metacognitivas de autoavaliação da aprendizagem. Constataram, também, que os alunos do sexo feminino apresentaram médias superiores nas subescalas que avaliavam as estratégias de planejamento e organização da aprendizagem.

Testa e Freitas (2005) realizaram um estudo acerca da autorregulação entre estudantes de Administração, Serviço Social, Sistemas de Informação e Análise de Sistemas. Aplicaram uma escala do tipo Likert de concordância de cinco pontos, com onze itens que avaliavam a percepção do estudante sobre sua gestão do ambiente de aprendizagem e do tempo. Neste estudo, constataram quatro perfis diferentes no curso de Administração, a saber: baixa capacidade de regulação do tempo e média regulação do ambiente e da prorrogação acadêmica; dificuldade geral de autorregulação da aprendizagem; boa capacidade para gerenciar tempo e ambiente de aprendizagem e média capacidade da prorrogação da gratificação; boa capacidade de regulação do tempo e baixa de gestão do ambiente. Os autores destacaram que os estudantes de Administração que se perceberam como mais autorregulados afirmaram ter notas superiores às dos seus colegas, enquanto aqueles pertencentes ao grupo que se percebe como de menor autorregulação do estudo declararam ter notas mais baixas. Ainda neste estudo foi verificada diferença entre sexo, sendo que as mulheres relataram ter uma maior autorregulação da gestão de seu tempo de estudo do que os homens. Os autores verificaram diferenças estatisticamente significativas para gestão de tempo entre os estudantes de Serviço Social e os demais cursos. Esses universitários informaram ter menos conteúdo de estudo atrasado do que os universitários dos outros cursos pesquisados porque estudavam com maior regularidade. Os estudantes de Sistemas de Informação foram os que revelaram estudar apenas nas vésperas das avaliações.

Núñez, Solano, Gonzáles-Pienda e Rosário (2006) realizaram uma pesquisa com 90 universitários portugueses para avaliar se a percepção do estudante sobre o seu processo de autorregulação da aprendizagem varia em função do conhecimento que ele tem acerca das estratégias autorregulatórias e técnicas de estudo. Os autores aplicaram uma escala de autorregulação como pré e pós-teste para avaliarem uma intervenção realizada com os alunos sobre estratégias e técnicas de estudo com duração total de 28 horas, distribuídas em seis sessões. Os resultados indicaram que, quando se aumenta o conhecimento sobre as estratégias menos observáveis (e.g., metacognitivas, motivacional, gestão de recursos de tempo e esforço), as medidas de autorrelato são afetadas positivamente; mas o mesmo não acontece em relação às estratégias cognitivas e de regulação de contextos de estudo (e.g. material, ambiente físico), uma vez que estas são mais amplamente conhecidas pelos universitários. 
A fim de analisar as estratégias de autorregulação de universitários das áreas de exatas e humanas, Ribeiro e Silva (2007) aplicaram a escala Comportamentos e hábitos de Estudo e Aprendizagem ( $\mathrm{CHE}$ ), que avalia cinco aspectos da autorregulação, a saber: estratégias cognitivas de transformação e manipulação da informação; organização e planejamento de rotinas; gestão e monitoramento; aquisição e seleção da informação; e reforço motivacional. Participaram 518 estudantes portugueses dos cursos de Física, Química, Engenharia Civil, Letras e Relações Internacionais. Estes foram distribuídos em diferentes grupos por etapas da graduação (inicial, intermediária e final). Os resultados mostraram que há correlação estatisticamente significativa entre as estratégias cognitivas e metacognitivas de gestão e monitoramento, mas não revelaram tais correlações entre reforço motivacional e estratégias de planejamento e monitoramento. Verificou-se que a percepção dos alunos acerca da sua autorregulação não apresentou diferenças estatisticamente significativas quanto à etapa de ensino e área, constatando-se apenas que os estudantes do curso de Letras se consideravam mais autorregulados que os demais.

Rosário e cols. (2007) realizaram uma pesquisa com o objetivo de constatar a eficácia de um programa de intervenção para melhorar as competências em autorregulação em estudantes ingressantes no Ensino Superior. Participaram desse estudo 108 estudantes de uma universidade espanhola, divididos em dois grupos: grupo-controle e grupo experimental. Como pré-teste e pós-teste utilizaram cinco instrumentos de avaliação de estratégias de autorregulação e de estilos de aprendizagem. A intervenção foi realizada em seis sessões com duração de uma hora sobre estratégias e organização da aprendizagem. Encontraram-se diferenças estatisticamente significativas entre os grupos experimental e controle no tocante ao conhecimento declarativo sobre estratégias de aprendizagem, ao estilo de aprendizagem preferencial (diminuição do uso de aprendizagem superficial pelo grupo experimental) e à qualidade de respostas relacionadas com a análise de tarefas de aprendizagem. Esses resultados indicam a eficácia do programa no ensino e desenvolvimento das estratégias de aprendizagem e reforçam os estudos internacionais sobre a importância de se trabalharem as competências de autorregulação da aprendizagem em universitários, visando desenvolver-lhes a habilidade para aprender a aprender.

Os estudos anteriormente descritos mostram a necessidade de se avaliarem as estratégias autorreguladas na aprendizagem e atividade de estudo. Ao lado disso, permitem constatar que o autorrelato é uma ferramenta útil para essa avaliação, desde que os estudantes se tornem mais conscientes dos aspectos menos observáveis e explicáveis de seu comportamento para que possam melhor planejar, monitorar e avaliar seu estudo. Nesse sentido, este estudo avaliou a percepção dos estudantes do Ensino Superior acerca das estratégias de autorregulação de estudo (planejamento, monitoramento e autoavaliação), considerando a caracterização das variáveis sexo, curso e idade para a amostra escolhida, tendo-se por perspectiva teórica o modelo de autorregulação de Zimmerman (2001; 2013).

\section{Método}

\section{Participantes}

Participaram deste estudo 126 estudantes universitários da área de Ciências Exatas de uma faculdade particular do Sul de Minas Gerais, dos quais $81 \%$ eram do sexo masculino. As idades variaram de 17 a 48 anos, com a média de $24,65(D P=3,54)$. Todos os estudantes estavam matriculados no $1^{\circ}$ semestre dos seguintes cursos: Engenharia de Produção (45,2\%), Engenharia de Controle e Automação (38,9\%), e Análise e Desenvolvimento de Sistemas (15,9\%).

\section{Instrumento}

Escala de Competência em Estudo - ECE-Sup (C\&T) (Almeida, \& Joly, 2009)

A escala objetiva avaliar os métodos de estudo e as abordagens referentes à aprendizagem dos estudantes no Ensino Superior. É composta por 19 itens com um formato de resposta de tipo Likert com quatro pontos - de quase nunca (1 ponto) até quase sempre (4 pontos) -, facultando-se ainda a possibilidade de não resposta ou "Não se aplica". Os itens agrupam-se em três fatores. O fator 1, Comportamentos Estratégicos de Planejamento, compreende nove itens, que se referem a decisões prévias relativas à forma como o estudante organiza o seu estudo - por exemplo, o item "Organizo um cronograma para estudar o conteúdo das disciplinas". O fator 2, Comportamentos Estratégicos de Monitoramento, que objetiva aferir os comportamentos relativos ao automonitoramento pela auto-observação do desempenho durante a execução de atividades de estudo, compõe-se de cinco itens, que podem ser exemplificados pelo item "Se facilitar o meu entendimento, estudo com os colegas". No fator 3, Comportamentos Estratégicos de Autoavaliação, os cinco itens reportam-se a comportamentos e a preocupações dos alunos no sentido de viabilizarem um estudo competente e bem-sucedido, sendo por isso dependentes da existência de uma autorreflexão do estudante acerca de seu desempenho, que pode ser feita anterior, concomitante ou posteriormente ao ato de estudar, como destacado pelo item "Depois de estudar, seleciono as fórmulas a serem utilizadas". A aplicação do instrumento é coletiva e dura, em média, 20 minutos.

O estudo de Almeida e Joly (2009) revela as qualidades pesicométricas da escala para o Brasil. A análise fatorial exploratória revelou que os itens se agruparam nos três fatores, revelando cargas fatoriais bastante altas, que variaram de 0,44 a 0,66, e comunalidade acima de $32 \%$. Os três fatores explicam $44,83 \%$ da variância da escala, sendo que o fator 1 explica 19,3\% da variância, o fator 2 explica $14,22 \%$ e o fator 3 explica $11,31 \%$ da variância. A análise 
da precisão foi realizada por meio da consistência interna dos itens (Alfa de Cronbach) para cada um dos três fatores. Constataram-se bons índices de precisão para a escala: no total, 0,81, de 0,79 para o fator 1 , de 0,76 para o fator $2 \mathrm{e}$ de 0,53 para o fator 3 . Deve-se considerar ainda que os resultados nas três dimensões se encontram correlacionados, como seria de esperar de acordo com o construto teórico da autorregulação. Os coeficientes de correlação produto versus momento de Pearson (para $p \leq 0,001$ ) foram 0,374 (fatores 1 e 2), 0,468 (fatores 1 e 3), e 0,352 (fatores 2 e 3). Considerando-se a correlação entre o escore total e cada um dos fatores, verificou-se correlação de 0,878 com o fator 1 , de 0,721 com o fator 2 e de 0,684 com o fator 3 . Piovezan (2013) confirmou a estrutura teórica e empírica da ECE (C\&T) pela análise fatorial confirmatória, o que lhe conferiu outra evidência de validade estrutural.

\section{Procedimentos}

Obtida a autorização da faculdade, os pesquisadores aplicaram o instrumento durante uma aula/palestra. Em todo o processo da pesquisa foram obedecidos os princípios éticos referentes à pesquisa envolvendo seres humanos estabelecidos pela Resolução 196/96 do Ministério da Saúde, que garante ao participante a confidencialidade das informações fornecidas, a manutenção de sua privacidade, o direito de interromper a participação no estudo em qualquer momento de sua realização e o seu consentimento livre e esclarecido.

\section{Resultados e discussão}

Considerando-se os objetivos deste estudo (avaliar a percepção de universitários acerca do uso de estratégias de autorregulação de estudo), os resultados obtidos com a escala utilizada foram analisados descritiva e inferencialmente a fim de obter dados para melhor caracterizar o construto. Assim, apresentam-se o desempenho geral dos estudantes e as diferenças de médias considerando-se as variáveis gênero, curso e idade.

A tabela 1 mostra os escores dos estudantes nos três fatores da escala e no conjunto dos seus itens (desempenho geral).

Tabela 1. Estatísticas descritivas da ECE-C\&T $(N=126)$

\begin{tabular}{lcccc}
\hline Escala & Mínimo & Máximo & Média & DP \\
\hline Planejamento & 08 & 34 & 23,78 & 5,25 \\
Monitoramento & 06 & 20 & 12,99 & 2,99 \\
Autoavaliação & 05 & 20 & 14,13 & 2,91 \\
\hline Escore total & 31 & 73 & 50,90 & 8,45 \\
\hline
\end{tabular}

Quanto à pontuação total para todos os fatores, constatou-se que a média geral dos estudantes foi superior ao ponto médio. Isto indica que os estudantes relataram que planejam, monitoram e avaliam seus comportamentos estratégicos de estudo para saber se estão sendo eficazes ou não na maneira de estudar os conteúdos acadêmicos. Nesse sentido, também foi constatado pelo escore geral que essa amostra apresentou média muito acima do ponto médio da escala, corroborando a pontuação dos fatores e demonstrando que os alunos recorrem a estratégias de autorregulação, como mostra a figura 1.

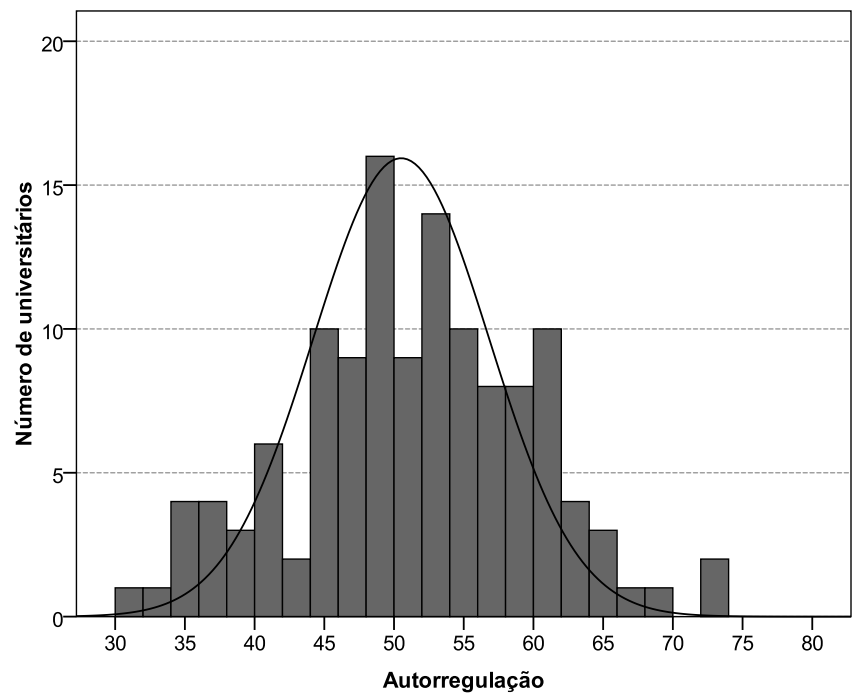

Figura 1. Histograma dos escores totais de autorregulação de 126 universitários.

A distribuição de frequência dos escores totais de autorregulação é quase simétrica e levemente mais achatada em relação à normal, podendo-se aceitar que a distribuição dos escores se ajusta à curva normal (K-S=0,054; $p=0,200)$. É importante ressaltar que o ponto médio da escala equivale a 38 pontos, e na distribuição dos dados observa-se que a pontuação 49 obteve maior frequência, ou seja, os alunos se percebem como autorreguladores de sua aprendizagem. Esta situação parece contrastar com a opinião de alguns autores - como Ribeiro e Silva, 2007; Rosário e Cols., 2007 -, os quais consideram que os estudantes universitários não possuem hábitos de estudo, como também não sabem selecionar as estratégias de aprendizagem adequadas para cada disciplina ou conteúdo escolar. Neste estudo, os alunos participantes consideram-se autorregulados, apesar de não ter sido possível identificar quão competentes são em seus estudos, tendo-se por referência Braun e cols. (2002).

Para verificar qual dos fatores da ECE-C\&T os estudantes mais utilizam, ou seja, quais as estratégias mais utilizadas no seu estudo, e verificar se a diferença por fator era significativa, realizou-se o Teste $t$ de Student simples pareando cada fator. Na tabela 2 estão apresentados os resultados dessas análises, mencionando as médias ponderadas, 
Tabela 2. Estatísticas descritivas e teste $t$ dos fatores da ECE-C\&T

\begin{tabular}{|c|c|c|c|}
\hline Fatores da ECE-C\&T & Média Ponderada* & $D P$ & Teste $t$ de Student \\
\hline Planejamento & 1,25 & 0,28 & \multirow{2}{*}{$t(125)=23,602 ; p<0,001$} \\
\hline Monitoramento & 0,68 & 0,01 & \\
\hline Planejamento & 1,25 & 0,28 & \multirow{2}{*}{$t(125)=22,586 ; p<0,001$} \\
\hline Autoavaliação & 0,74 & 0,01 & \\
\hline Monitoramento & 0,68 & 0,01 & \multirow{2}{*}{$t(125)=-3,417 ; p<0,001$} \\
\hline Auto-avaliação & 0,74 & 0,01 & \\
\hline
\end{tabular}

Tabela 3. Teste t dos fatores da ECE-C\&T em função do sexo.

\begin{tabular}{|c|c|c|c|c|c|c|}
\hline Fatores & Sexo & $M$ & $D P$ & $t$ & $d f$ & $p$ \\
\hline \multirow{2}{*}{ Planejamento } & Feminino & 26,17 & 4,66 & \multirow{2}{*}{2,533} & \multirow{2}{*}{124} & \multirow{2}{*}{0,013} \\
\hline & Masculino & 23,22 & 5,24 & & & \\
\hline \multirow{2}{*}{ Monitoramento } & Feminino & 13,13 & 3,14 & \multirow{2}{*}{0,241} & \multirow{2}{*}{124} & \multirow{2}{*}{0,810} \\
\hline & Masculino & 12,96 & 2,97 & & & \\
\hline \multirow{2}{*}{ Autoavaliação } & Feminino & 14,63 & 2,57 & \multirow{2}{*}{0,916} & \multirow{2}{*}{124} & \multirow{2}{*}{0,361} \\
\hline & Masculino & 14,02 & 2,98 & & & \\
\hline \multirow{2}{*}{ Escore total } & Feminino & 53,92 & 8,22 & \multirow{2}{*}{1,987} & \multirow{2}{*}{124} & \multirow{2}{*}{0,055} \\
\hline & Masculino & 50,20 & 8,38 & & & \\
\hline
\end{tabular}

pois o fator comportamentos estratégicos de planejamento apresenta número itens superior ao dos outros dois fatores.

Constatou-se que as estratégias de planejamento do estudo são as mais utilizadas pelos estudantes dessa amostra. Esta inclui, dentre outros comportamentos, anotações em sala de aula, organização de cronograma, horário e conteúdo de estudo, leitura adicional e realização de exercícios. Observa-se, também, que as estratégias menos utilizadas são as de monitoramento do estudo, as quais implicam em habilidades relativas à comparação entre uma situação de estudo anterior e uma posterior, considerando resultados obtidos versus empenho e engajamento no estudo. Cumpre levar em conta, quando se monitora o estudo, a discussão, o tempo e qualidade do estudo com colegas de classe. Cabe destacar que há diferenças estatisticamente significativas entre os três fatores (tabela 2), o que ratifica o estudo de Núñes e cols. (2006).

Constatado esse resultado, é necessário investigar o que leva os estudantes da área de Exatas a priorizarem o uso de uma estratégia para o estudo. Será que planejar como estudar e não monitorar possibilita a obtenção de bons resultados acadêmicos? Por que os estudantes, apesar de informarem que monitoram pouco seu processo de estudo, indicaram que se autoavaliam sobre seus resultados? Qual a relação que há entre os três comportamentos estratégicos? Ao lado disso, pode-se hipotetizar que quando o aluno aumenta o seu conhecimento sobre as estratégias de aprendizagem, aumenta também sua percepção de que a utiliza? Se assim for, os estudantes poderiam ampliar seus comportamentos estratégicos de estudo, caso eles possuíssem mais conhecimento acerca desses? A dimensão e implicação das respostas às questões estão imbricadas tanto no referencial teórico que as sustenta (Zimmerman, 2001; Braun, $\&$ cols., 2002) quanto no desenvolvimento de um processo investigativo que possibilite compreendê-las melhor (Núñez, \& cols., 2006; Ribeiro, \& Silva, 2007; Rosário, \& cols., 2007; Tavares, \& cols., 2003; Testa, \& Freitas, 2005).

Para verificar a diferença de estratégias de estudo em função do sexo realizou-se o Teste $t$ de Student. A tabela 3 apresenta os dados dessa análise.

Pode-se observar que no fator comportamentos estratégicos de planejamento e no escore total houve diferença estatisticamente significativa entre os sexos, sendo que as mulheres relataram utilizar mais estratégias de planejamen- 
to de estudo que os homens. Esse resultado corrobora estudos já citados (Tavares e cols., 2003; Testa \& Freitas, 2005), nos quais as mulheres relataram o uso mais frequente das estratégias de autorregulação de planejamento.

Quanto ao curso, não houve diferença estatisticamente significativa para nenhum fator da ECE-C\&T, Planejamento $(F[2,123]=0,453 ; p=0,637)$, Monitoramento $(F[2$, $123]=0,172 ; p=0,842)$ e Autoavaliação $(F[2,123]=0,089$; $p=0,915)$, assim como para o escore total da escala $(F[2$, $123]=0,417 ; p=0,660)$, de acordo com a análise de variância das médias de frequências de respostas aos itens por fator da escala (ANOVA). Esse dado ratifica Testa e Freitas (2005), que não encontraram diferença por curso na amostra por eles investigada; mas estes resultados diferem dos obtidos por Ribeiro e Silva (2007), que indicaram diferenças estatisticamente significativas para estudantes de um curso de Letras quanto às variáveis gestão e monitoramento e organização e planejamento de rotinas em relação aos demais cursos considerados pelos autores.

Para verificar a diferença de estratégias de estudo por idade, os estudantes foram agrupados em três faixas etárias. Na faixa 1 estavam os estudantes menores de 20 anos ( $N=41)$; na faixa 2, os de 21 a 25 anos $(N=38)$; e na faixa 3, os estudantes com mais de 26 anos de idade $(\mathrm{N}=47)$. Realizou-se uma ANOVA por faixa etária, mas não foram encontradas diferenças estatisticamente significativas para nenhum fator da ECE-C\&T, Planejamento $(F[2,123]=0,954$; $p=0,388)$, Monitoramento $(F[2,123]=0,304 ; p=0,738)$ e Autoavaliação $(F[2,123]=0,615 ; p=0,542)$ e para o Escore total $(F[2,123]=0,812 p=0,446)$. Cabe considerar que resultados semelhantes foram encontrados por Ribeiro e Silva (2007) no tocante à etapa escolar.

\section{Considerações finais}

De modo geral, os resultados ratificam a visão de aprendizagem centrada no aluno. Em assim sendo, pode-se afirmar que há uma mudança gradativa da visão da aprendizagem como mera aquisição de informação para uma orientação mais construtivista, na qual se valoriza a apropriação e o desenvolvimento de competências, resultando em maior autonomia dos alunos (Zimmerman, 2001). Em síntese, os resultados do presente estudo indicaram que planejar é o comportamento estratégico mais utilizado, segundo o relato dos universitários da amostra. Isto significa que estão mais presentes competências de autorregulação em termos de condutas de estudo e não tanto em termos mais cognitivos e metacognitivos. Pôde-se constatar, ainda, que as universitárias utilizam com maior frequência comportamentos estratégicos em geral e, especificamente, o planejamento. Não foram observadas diferenças estatisticamente significativas para a autorregulação do estudo em relação às variáveis curso e idade dos participantes. Tais resultados, específicos para a amostra investigada, atenderam aos objetivos a que se propôs a pesquisa de levantamento descrita. É indiscutível que, a partir deste estudo exploratório, possa-se ampliar e diversificar a amostra de participantes e realizar novas investigações em diferentes regiões brasileiras, a fim de dar maior robustez à validade dos resultados neste instrumento e permitir uma melhor caracterização dos comportamentos de autorregulação da aprendizagem e do estudo por parte dos estudantes universitários no Brasil.

Por outro lado, importa aprofundar, em pesquisas futuras, a relação entre comportamentos estratégicos de estudo e o desempenho acadêmico e entre motivação e autoeficácia, por exemplo. Ao mesmo tempo, importa estudar o impacto de variáveis sociais, culturais e do contexto educacional na autorregulação de estudo. Os resultados possibilitarão uma melhor identificação das variáveis relevantes em termos de aprendizagem e sucesso acadêmico. Neste sentido considera-se, de um lado, a diversidade socioeconômica, cultural e educacional e as habilidades cognitivas dos estudantes (Zimmerman, 2001), e de outro, o contexto de ensino e aprendizagem da universidade (Ribeiro, \& Silva, 2007; Freire, 2009). Todos estes aspetos são fundamentais quando as instituições e os professores pretendem intervir em prol da melhor aprendizagem e do sucesso acadêmico dos seus estudantes.

As análises realizadas permitem ampliar as informações anteriormente identificadas por outros estudos realizados com universitários por meio de comparação entre os resultados, ratificando-os ou refutando-os. Assim, atende-se à premissa científica da pesquisa de levantamento, que busca caracterizar as variáveis presentes num dado construto. A partir dessas evidências cumulativas, é necessário fazer outras investigações, de caráter quase-experimental e experimental, associadas a pesquisas de cunho qualitativo (Almeida, \& Freire, 2008).

\section{Referências}

Almeida, L. S., \& Freire, T. (2008). Metodologia da investigação em Psicologia e Educação. 5a Ed. Braga: Psiquilíbrios Edições.

Almeida, L. S., \& Joly, M. C. R. A. (2009). Construção de instrumentos para Ensino Superior: estudos em Portugal/Brasil/Espanha/ Moçambique. Pesquisa Transcultural em desenvolvimento. Braga: Universidade do Minho (Portugal)/ Itatiba: Universidade São Francisco (Brasil).

Braun, H. J., Jackson, D. N., \& Wiley, D. E. (2002). The role of Constructs in Psychological and Educational Measurement. Hillsdale, NJ: Lawrence Erlbaum.

Freire, L. G. L. (2009). Auto-regulação da aprendizagem. Ciências \& Cognição, 14(2), 276-286.

Núñez, J.C., Solano, P., Gonzáles-Pienda, J.A., \& Rosário P. (2006). Evaluación de los procesos de autorregulación mediante autoinforme. Psicothema, 18(3), 353-358.

Rosário, P., Mourão, R., Núñez, J. C., Gonzálos-Pienda, J., Solano, P., \& Valle, A. (2007). Eficacia de un programa instruccional para la 
mejora de procesos y estrategias de aprendizaje en la enseñaza superior. Psicothema, 19(3), 422-427.

Ribeiro, I. S., \& Silva, C. F. (2007). Auto-regulação: Diferenças em função do ano e área em alunos universitários. Psicologia: Teoria e Pesquisa, 22(4), 443-448.

Piovezan, N. M. (2013). Estudos psicométricos com Escala de Competência de Estudo: avaliação da Autorregulação da Aprendizagem em universitários. Tese de Doutorado, Universidade São Francisco, Itatiba, SP.

Tavares, J., Bessa, J., Almeida, L. S., Medeiros, M. T., Peixoto, E., Ferreira, J. A. (2003). Atitudes e estratégias de aprendizagem em estudantes do Ensino Superior: Estudo na Universidade dos Açores. Análise Psicológica, 4(21), 475-484.
Testa, M. G., \& Freitas, H. (2005). Auto-regulação da Aprendizagem: Analisando o perfil do estudante de Administração. Em XXIX Encontro Nacional da ANPAD (ENANEAD). Anais do XXIX Encontro Nacional da ANPAD (ENANEAD). Brasília: ANPAD.

Zimmerman, B. J. (2001). Theories of self-regulated learning and academic achievement: An overview and analysis. Em B. J. Zimmerman, \& D. H. Schunk (Orgs.), Self-regulated learning and academic achievement: Theoretical perspectives (2nd ed., pp. 1-37). Mahwah, NJ: Lawrence Erlbaum.

Zimmerman, B. J. (2013). From Cognitive Modeling to Self-Regulation: A Social Cognitive Career Path. Educational Psychologist, 48 (3), 135-147.

\section{Sobre os autores}

Maria Cristina Rodrigues Azevedo Joly (mcrisjoly@gmail.com)

Doutora em Psicologia da Aprendizagem e Desenvolvimento Humano - USP

Diego Vinícius da Silva (vinicius.diego@ymail.com)

Mestre em Psicologia - USF

Carla Fernanda Ferreira-Rodrigues (carlafernandafr@gmail.com)

Mestre em Psicologia - UFRN

Juliana Maximila de Paula Bueno (julianampbueno@hotmail.com)

Mestre em Psicologia - USF

Leandro Silva Almeida (leandro@ie.uminho.pt)

Doutor em Psicologia da Educação pela Universidade do Porto 